\title{
The investigation of AIGaN/GaN HEMT failure mechanisms under different temperature conditions
}

\author{
Chunsheng Guo ${ }^{1, a}$, Yunxiang Ren ${ }^{1, b}$, Li Gao ${ }^{2}$, Hui Zhu ${ }^{1}$ and Shiwei $\mathrm{Li}^{1}$ \\ ${ }^{1}$ College of Electronic Information and Control Engineering, Beijing University of Technology, \\ 100124 \\ ${ }^{2}$ China Electronics Standardization Institute, 100176 \\ aemail: guocs@bjut.edu.cn, bemail: ren959551@163.com
}

Keywords: AIGaN/GaN HEMT; the Schottky barrier; junction temperature

\begin{abstract}
To explore degradation law and failure mechanisms of AlGaN/GaN HEMT under different temperature conditions, the step-temperature stress experiments are carried out. The results show that the drain-source current decreases with the aging time when the junction temperature range from $139{ }^{\circ} \mathrm{C}$ to $200^{\circ} \mathrm{C}$; while the drain-source current increases with the aging time when the junction temperature range from $200^{\circ} \mathrm{C}$ to $352^{\circ} \mathrm{C}$. When the junction temperature is less than $200^{\circ} \mathrm{C}$, the AlGaN ionized donor atoms cause the Schottky barrier height of the AlGaN/GaN HEMT device to rise; and when the junction temperature is higher than $200^{\circ} \mathrm{C}$, the diffusion of impurities oxygen in surface causes the Schottky barrier height of the AlGaN/GaN HEMT device to fall. The barrier height can affect the threshold voltage which leads to the change of drain-source current. Therefore, the degradation of the drain-source current is caused by the change of the Schottky barrier height.
\end{abstract}

\section{Introduction}

The GaN materials have many advantages including large band gap, high electron saturation velocity, good thermal conductivity, and etc. They have shown excellent performance in high power and high temperature applications. The AlGaN/GaN high electron mobility transistors (HEMTs) are representative of the GaN-based microelectronic devices, which can be widely used in energy exploration, radar systems and anti-missile systems. However, the performance of GaN HEMTs are affected by many factors, such as the production processes, complex structures, special polarization effects, high electric field stress and etc. Therefore, the reliability problem of AlGaN/GaN HEMTs have become a hot issue of current research, especially for the device working under high temperature or high electric field conditions [1][2].

The degradation of the GaN HEMTs have been investigated extensively. Pazirandeh et al. found that the gate voltage of GaN HEMTs increased gradually with the aging time under the constant voltage and power when the case temperature was $150^{\circ} \mathrm{C}$ [3].Dammann et al. found that the leakage current of GaN HEMTs decreased first and then increased with the aging time under different surface temperature using the drain-current step-stress tests. But the explanation of the phenomenon was limited [4]. Yang Zhen et al. considered that the degradation of saturation drain current is caused by the positive shift in $\mathrm{V}_{\mathrm{T}}$ under semi-on DC stress [5]. Through the step temperature stress experiments under high electric field, Wu and his colleagues found that the maximum drain current remained unchanged at low temperature, whereas the current rapidly decreased with the aging time under high temperature. The reason was because the stressed samples developed pits and trenches to trap carriers under the gate edge on the drain side of the device [6]. From the present study, there is no consistent result on the degradation process of the GaN HEMTs, nor the failure mechanism is explained in detail.

To explore failure mechanisms, this paper studied the degradation process, and the corresponding mechanism of GaN HEMTs with aging time under different temperature conditions. The experimental GaN HEMT devices were loaded rated power and step temperature stress. Based on the consistency of failure mechanisms at different temperature [7], the forward Schottky barrier 
height was measured in order to obtain the failure mechanisms of GaN HEMTs under different temperature.

\section{Experiment}

The GaN HEMT devices used in this study were produced by Cree. The testing took place in a thermostatic platform. The devices were stressed by the step temperature at $\mathrm{V}_{\mathrm{DS}}=28 \mathrm{~V}$ and $\mathrm{I}_{\mathrm{DS}}=500 \mathrm{~mA}$. The initial temperature of the platform was set as $20^{\circ} \mathrm{C}$, and the temperature was raised by $25^{\circ} \mathrm{C}$ every 24 hours. After working 24 hours at each temperature, the devices were removed from the platform. Then, the samples were cooled to room temperature and measured.

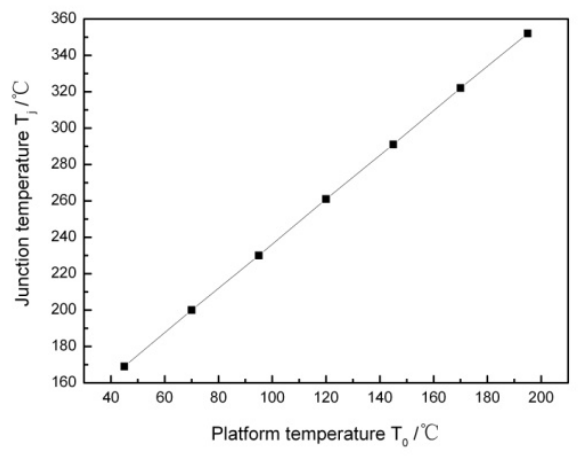

Fig. 1 The function of the junction temperature and platform temperature

To prevent the self-excited oscillation of the devices, this paper designed the protection circuit. During the experiments, the junction temperature can be estimated through the real-time monitoring case temperature of the devices. Based on the correlation between the track recorded case temperature $T_{C}$ and the platform temperature $T_{0}$, the expression of $T_{C}$ can be determined by: $\mathrm{T}_{\mathrm{C}}=0.986 \times \mathrm{T}_{0}+41.57$. Through the infrared measuring experiments, the correlation between the junction temperature $T_{j}$ and the case temperature $T_{C}$ was determined by: $T_{j}=1.24 \times T c+63.01$. So the relationship between the junction temperature and the platform temperature is derived to be: $\mathrm{T}_{\mathrm{j}}=1.22 \times \mathrm{T}_{0}+114.56$, as it is shown in Fig. 1. The output characteristic curves and the forward Schottky characteristic curves were measured by the Agilent B1500.

\section{Results and Discussion}

The output characteristic curves $\left(\mathrm{V}_{\mathrm{GS}}=-2.2 \mathrm{~V}\right)$ of the GaN HEMT under the step-temperature stress experiments are displayed in Fig. 2. It shows that the drain-source current $\mathrm{I}_{\mathrm{DS}}$ decreases with the aging time when the junction temperature is below $200^{\circ} \mathrm{C}$. However, when the junction temperature increases above $200^{\circ} \mathrm{C}$, the $\mathrm{I}_{\mathrm{DS}}$ begin to increase with the aging time. The degradation of $I_{D S}$ with the aging time under $V_{D S}=13 \mathrm{~V}$ is shown in Fig. 3, where the solid line represents the maximum drain source current $\mathrm{I}_{\mathrm{DSM}}$ as a function of the aging time, and the hollow line represents the normalized degradation ratio of $\mathrm{I}_{\mathrm{DSM}}$. 


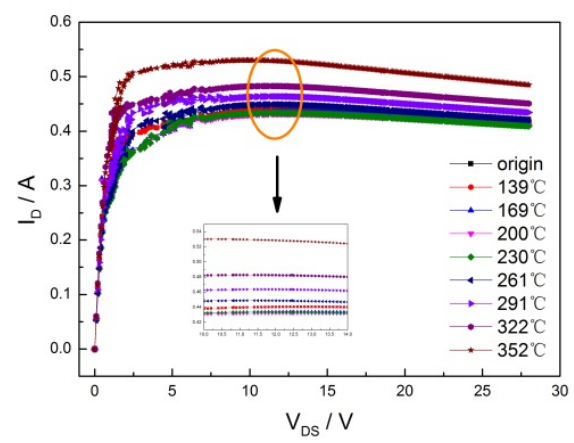

Fig. 2 Output characteristic curves after different temperature stress

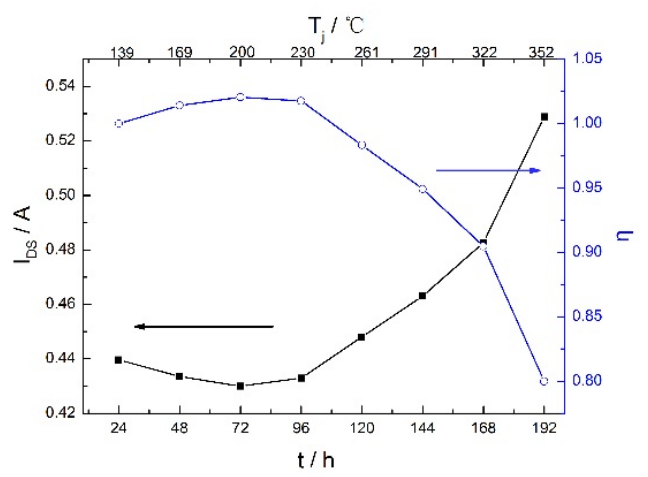

Fig. 3 The degradation of the drain current with aging time

When the junction temperature is below $200{ }^{\circ}$ he $\mathrm{I}_{\mathrm{DSM}}$ of the GaN HEMT decreases with the aging time. After working at the junction temperature of $200^{\circ} \mathrm{C}$ for $24 \mathrm{~h}$, the degradation ratio of $\mathrm{I}_{\mathrm{DSM}}$ is $3 \%$. To avoid the measurement error, a group of GaN HEMT devices standing at the room temperature are compared, with the degradation ratio in $\mathrm{I}_{\mathrm{DSM}}$ less than $0.8 \%$. This indicates that the degradation of $\mathrm{I}_{\mathrm{DSM}}$ working at the junction temperature below $200^{\circ} \mathrm{C}$ is not caused by the measurement error. When the junction temperature is above $200^{\circ} \mathrm{C}$, the $\mathrm{I}_{\mathrm{DSM}}$ increases with the aging time. When the device works at the junction temperature of $352^{\circ} \mathrm{C}$ for $24 \mathrm{~h}$, the changes of $\mathrm{I}_{\mathrm{DSM}}$ are more than $20 \%$. According to the failure criterion, the experiments are finished.

The failure mechanisms of $I_{D S}$ under different junction temperature conditions are analyzed as follows. In the saturation region, the equation of the drain current is:

$I_{\text {Dsat }}=\frac{\kappa}{2}\left(V_{G}-V_{T}\right)^{2}$

where $\kappa$ is a constant, which is related to the electron mobility. $V_{T}$ is threshold voltage. It is known that the $\mathrm{I}_{\mathrm{DS}}$ depends on the constant $\kappa$, gate voltage $V_{G}$ and threshold voltage $V_{T}$. The gate voltage $V_{G}$ keeps constant $\left(\mathrm{V}_{\mathrm{GS}}=-2.2 \mathrm{~V}\right)$ under the same electrical stress test condition. For HEMT devices, the scattering mechanism is mainly acoustic wave scattering and the test environment is room temperature, so the electron mobility is not changed. As a result, the $\mathrm{I}_{\mathrm{DS}}$ mainly depend on the threshold voltage $V_{T}$ under the room temperature. Due to the value of $V_{T}$ and $V_{G}$ are negative, the $\mathrm{I}_{\mathrm{DS}}$ will increase with the negative shift of $V_{T}$. As is shown in Fig. 4, the variation of the threshold voltage corresponds exactly with the degradation of the $\mathrm{I}_{\mathrm{DS}}$. 


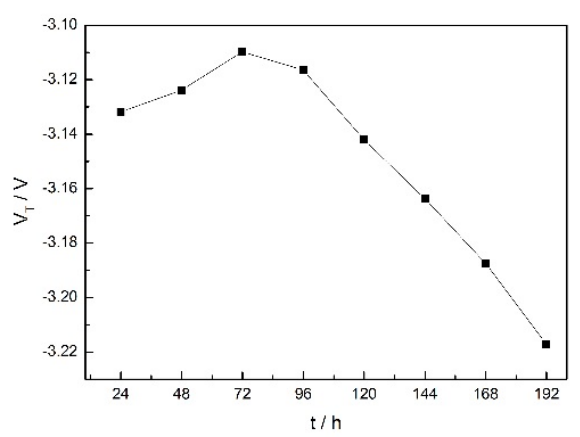

Fig. 4 The degradation of the threshold voltage with aging time

Because the threshold voltage is determined by the AlGaN / GaN HEMT Schottky barrier height [8], we analyze the changes in the barrier height under different conditions. It shows in Fig. 3 that the degradation of $\mathrm{I}_{\mathrm{DSM}}$ with aging time is non-monotonic, implying that there should exist different failure mechanisms in the total degradation. In different temperature ranges, the main degradation mechanisms are different. When the junction temperature is below $200^{\circ} \mathrm{C}$, some of the $\mathrm{AlGaN}$ donor atoms will ionize. The free electrons from the ionization can drift to the quantum well so that the AlGaN / GaN barrier depletion region widens, and the barrier height increases [9]. The rise of the barrier height leads to the positive shift of $V_{T}$. Through the XPS analysis, it is found that the AlGaN have adsorbed some oxygen impurities [10]. When the junction temperature is above $200^{\circ} \mathrm{C}$, the oxygen can easily diffuse into the AlGaN barrier layer and combine with $\mathrm{Al}$, resulting in the $\mathrm{Al}$ $\mathrm{O}$ bond. This is the main reason of the reduction of the AlGaN / GaN Schottky barrier height [11], which causes the negative shift of $V_{T}$.

\section{Verification}

To verify the changes of the barrier height, the forward Schottky characteristic curves are measured. The quantitative changes in the barrier height can be obtained according to the current formula of the classical hot electrons emission theory:

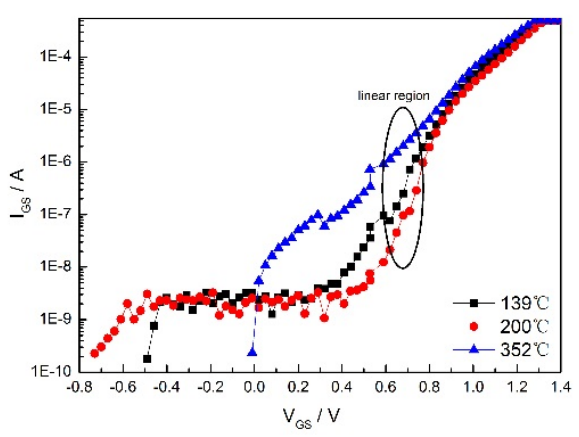

Fig. 5 The forward Schottky I-V characteristic curves in semi-logarithmic $\mathrm{J}=A^{*} T^{2} \exp \left(-\frac{q \varphi}{k T}\right)\left[\exp \left(\frac{q V}{k T}\right)-1\right.$

where $\mathrm{A} *$ (equal to $34.2 \mathrm{~A} / \mathrm{cm}^{2} * \mathrm{~K}^{2}$ at room temperature) is the Richardson effective constant, $\varphi$ the barrier height and $\mathrm{T}$ the temperature, respectively.

The current of the AlGaN / GaN Schottky contact can be expressed as:

$\mathrm{I}=\mathrm{A} A^{*} T^{2} \exp \left(-\frac{q \varphi}{k T}\right)\left[\exp \left(\frac{q V}{n k T}\right)-1\right]$

where A(equal to $1.39 \times 10^{-6} \mathrm{~cm}^{2}$ ) is the Schottky contact area. The equation (2) can be converted by the logarithmic transformation:

$\lg \mathrm{I}=\frac{\lg e}{k T} * \frac{q}{n} * V+\lg A A^{*} T^{2}+\lg e * \frac{-q \varphi}{k T}$ 
Therefore, the forward Schottky I-V characteristic curves in semi-logarithmic is shown in Fig. 5. In the linear region of the middle part in the semi-logarithmic curves, the main mechanism of the forward Schottky current is thermionic emission. Then the straight lines can be fitted in the linear region. The intersection with the vertical axis can be expressed as:

Intercept $=\lg A^{*} A T^{2}+\lg \mathrm{e} * \frac{-q \varphi}{k T}$

So the barrier height can be determined as follows:

$\varphi=\frac{k T}{q \lg e} * \lg \frac{A^{*} A T^{2}}{\text { Intercept }}$

The expression (5) indicates that the Schottky barrier height decreases as the increase of the intercept. Fig. 5 shows that the intercept will decrease with the aging time when the junction temperature is below $200^{\circ} \mathrm{C}$, resulting in the increase of the barrier height. When the junction temperature is above $200^{\circ} \mathrm{C}$, the intercept will increase with the aging time and hence, the decrease of the barrier height. The barrier height is obtained and listed in Table 1. When the junction temperature is below $200^{\circ} \mathrm{C}$, the $I_{D S}$ will decrease because the barrier height of the GaN HEMT increases, resulting in the positive shift of $V_{T}$. When the junction temperature is above $200^{\circ} \mathrm{C}, \mathrm{I}_{\mathrm{DS}}$ will increase because the barrier height of the GaN HEMT decreases, resulting in the negative shift of $V_{T}$.

Table 1 the barrier height after different temperature stress

\begin{tabular}{ll}
\hline \hline Junction temperature $/{ }^{\circ} \mathrm{C}$ & Barrier height $/ \mathrm{eV}$ \\
\hline 139 & 1.11 \\
169 & 1.15 \\
200 & 1.22 \\
230 & 1.17 \\
261 & 1.08 \\
291 & 0.99 \\
322 & 0.93 \\
352 & 0.81 \\
\hline \hline
\end{tabular}

\section{Conclusion}

In the GaN HEMT step-temperature stress experiments, the evolution of drain source current with the aging time shows the non-monotonic degradation characteristics. The current decreases under low temperature first, then it increases under high temperature. Considering the degradation process, the forward Schottky characteristics curves of the GaN HEMT are measured in order to verify the changes of the Schottky barrier height. It is found that when the junction temperature is less than 200 tee, ionization of AlGaN donor atoms leads AlGaN/GaN barrier height to rise, resulting in the positive shift of $V_{T}$. When the junction temperature rises above $200^{\circ} \mathrm{C}$, the diffusion of oxygen impurities in surface causes the Schottky barrier height of the GaN HEMT device to fall, thus, resulting in the negative shift of $V_{T}$. Therefore, the degradation of the drain-source current is caused by the change of the Schottky barrier height. 


\section{Reference}

[1] J. A. del Alamo, J. Joh. GaN HEMT reliability. Microelectronics Reliability, 2009, 49(9): 1200

[2] D. Marcon, T. Kauerauf, F. Medjdoub, et al. A comprehensive reliability investigation of the voltage, temperature and device geometry-dependence of the gate degradation on state-of-theartAlGaN/GaN-on-Si HEMTs. IEEE IEDM, 2010: 20

[3] R. Pazirandeh, J. Würfl, G. Trankle, et al. Determination ofAlGaN/GaN HEMT reliability by monitoring $I_{\text {DSs }}$. Microelectron Reliab, 2010, 50: 763

[4] Yun-HsiangWang, Yung C Liang, S Samudra, et al. Modelling temperature dependence on $\mathrm{AlGaN} / \mathrm{GaN}$ power HEMT device Characteristics. Semiconductor science and technology, 2013, 28(5): 208

[5] Yang Zhen, Wang Jinyan, Xu Zhe, et al. Analysis of AlGaN/GaN high electron mobility transistors failure mechanism under semi-on DC stress. Journal of Semiconductors, 2014, 35(1): 014007

[6] Y. Wu, C.-Y. Chen, J. A. del Alamo, et al. Electrical and structural degradation ofAlGaN/GaN high electron mobility transistors under high-power and high-temperature Direct Current stress. Journal of Applied Physics, 2015, 11(7): 025707

[7] Guo Chunsheng, Zhang Yanfeng, Wan Ning, et al. Identifying the failure mechanism in accelerated life tests by two-parameter lognormal distributions. Journal of Semiconductors, 2014, 35(8): 084010

[8] Zhihong Feng, Shengyin Xie, Rui Zhou, et al. High-performance enhanced-mode AlGaN/GaN HEMT. Journal of Semiconductors, 2010, 31(8): 45

[9] Zhang Xiaoling, Xie Xuesong, Lv Changzhi, et al. The temperature characteristics of the schottky barrier in the Ni/AlGaN/GaN structure, Beijing University of technology, 2008, 34(4): 153 (in Chinese)

[10] S.K. Hong, K.H. Shim, J.W. Yang, et al. Reduced gate leakage current in AlGaN/GaN HEMT by oxygen passivation of AlGaN surface. Electronics Letters, 2008, 44(18): 1183

[11] Martin Kuball, Milan Tapajna, Richard J.T. Simms, et al. AlGaN/GaN HEMT device reliability and degradation evolution: Importance of diffusion processes. Microelectronics Reliability, 2011, 51(20): 195 